NONSTANDARD

METHODS FOR

STOCHASTIC

FLUID MECHANICS 
This page is intentionally left blank 
Series on Advances in Mathematics for Applied Sciences - Vol. 27
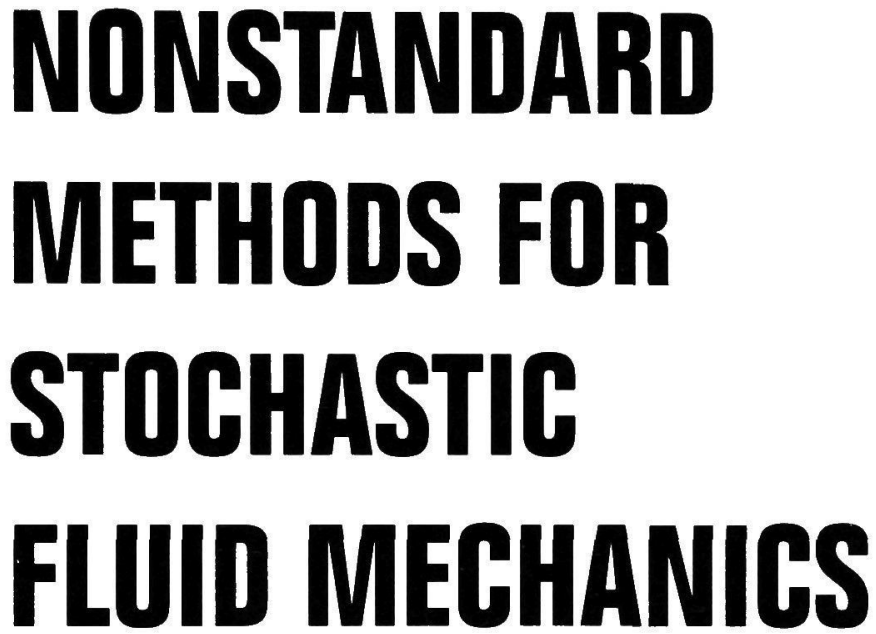

\section{Marek Capiński}

Institute of Mathematics, Jagiellonian University, Poland

Nigel J. Cutland

School of Mathematics, The University of Hull, UK 


\section{Published by}

World Scientific Publishing Co. Pte. Ltd.

P O Box 128, Farrer Road, Singapore 9128

USA office: Suite 1B, 1060 Main Street, River Edge, NJ 07661

UK office: 57 Shelton Street, Covent Garden, London WC2H 9HE

\section{Library of Congress Cataloging-in-Publication Data}

\section{Capiński, Marek, 1951-}

Nonstandard methods for stochastic fluid mechanics / Marek Capiński, Nigel J. Cutland.

p. $\quad \mathrm{cm}$. -. (Series on advances in mathematics for applied sciences; vol. 27) Includes bibliographical references and index.

ISBN 9810217102

1. Fluid mechanics. 2. Nonstandard mathematical analysis.

3. Stochastic processes. I. Cutland, Nigel. II. Title.

III. Series.

QA901.C36 1995

$532 \cdot .58--\mathrm{dc} 20$

Copyright $(1) 1995$ by World Scientific Publishing Co. Pte. Ltd.

All rights reserved. This book, or parts thereof, may not be reproduced in any form or by any means, electronic or mechanical, including photocopying, recording or any information storage and retrieval system now known or to be invented, without written permission from the Publisher.

For photocopying of material in this volume, please pay a copying fee through the Copyright Clearance Center, Inc., 27 Congress Street, Salem, MA 01970, USA. 
To Ewa and Mary $\odot \odot$

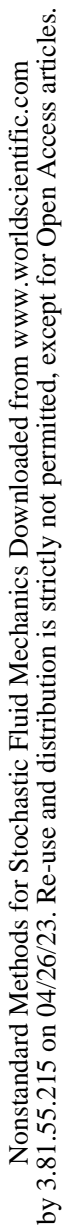


This page is intentionally left blank 


\section{Preface}

This work is concerned with new techniques and new results for equations of hydromechanics with stochastic perturbations. The emphasis is mainly on the introduction of random features into the classical Navier-Stokes equations:

$$
\begin{gathered}
\frac{\partial u}{\partial t}-\nu \Delta u+\langle u, \nabla\rangle u=f-\nabla p \\
\operatorname{div} u=0 \\
u(0)=u_{0}
\end{gathered}
$$

for the velocity $u=\left(u^{1}, \ldots, u^{d}\right)$ of an incompressible fluid in a bounded domain $D \subseteq \mathbb{R}^{d}$ where $d \leq 3$, subject to external forces $f$.

The stochastic perturbations considered are of two kinds: first we allow randomness in the initial condition $u_{0}$, which gives rise to the notion of statistical solution developed by Foias. Secondly we allow the force to be random, and this is modelled mathematically by the addition of a term involving white noise, in the form of a stochastic differential. The resulting equation in its most general form is then the following stochastic partial differential equation:

$$
\begin{gathered}
\frac{\partial u}{\partial t}-\nu \Delta u+\langle u, \nabla\rangle u=f(t, u)+g(t, u) \frac{d w}{d t}-\nabla p \\
\operatorname{div} u=0 \\
u(0)=u_{0}
\end{gathered}
$$

where $u_{0}$ is a random variable independent of $w$ and $\frac{d w}{d t}$ stands for white noise.

The new techniques use tools from nonstandard analysis, but the results obtained are entirely standard - they are formulated in the conventional Hilbert space setting of the Navier-Stokes equations, as developed by Leray, Temam, and others. Nonstandard techniques for these equations have been developed in a series of papers by the authors (see the bibliography). This book is designed to provide a unified account of these developments together with a self-contained introduction to the basic nonstandard tools that are needed for a detailed understanding of the ideas. In addition, there are some new results presented here for the first time. 


\section{Nonstandard Methodology}

At the heart of nonstandard analysis is the construction of an extension ${ }^{*} \mathbb{R}$ (the hyperreals) of the set of real numbers, which contains infinitesimals as well as infinite numbers. These include infinite natural numbers in the sense of ${ }^{*} \mathbb{R}$, which are called hyperfinite. This allows the construction of a projection $\mathbf{H}_{N}$, where $N$ is an infinite natural number and $\mathbf{H}$ is the fundamental Hilbert space in which the Navier-Stokes equations are set. Then $\mathbf{H}_{N}$ has two important features. First, it is a hyperfinite-dimensional projection and has all the properties of standard finite dimensional projections $\mathbf{H}_{n}$, using the fundamental transfer principle of nonstandard analysis. Secondly, there is a natural "projection" from $\mathbf{H}_{N}$ to $\mathbf{H}$ called the standard part mapping.

Using these ideas the essence of our approach to the solution of the Navier-Stokes equations is as follows: first formulate the approximation to the equations in $\mathbf{H}_{N}-$ this is the Galerkin approximation in dimension $N$, which can be solved easily using the transfer principle. Then use the standard part mapping to project this solution into $\mathbf{H}$, and show that it gives a solution to the standard equations. It is at this stage that the nonstandard approach is especially convenient, since it circumvents the need to establish convergence of Galerkin approximations, which is achieved in the standard approach by the use of various specialised compactness theorems.

For stochastic equations, nonstandard analysis provides the powerful tool of Loeb measure spaces. These spaces are very rich and carry additional structure that makes them tractable. They are used in two main ways here. First, any random initial condition $u_{0}$ can be represented by a Loeb measure on $\mathbf{H}_{N}$, and combined with the approach outlined in the previous paragraph this gives an elementary construction of statistical solutions. Second, when carrying out the programme described above for the general stochastic equations, the projection of the (nonstandard) stochastic solution in $\mathbf{H}_{N}$ to the space $\mathbf{H}$ gives a standard stochastic process carried on a Loeb probability space. Here it is the richness of the Loeb space that facilitates the construction: by contrast, in a standard approach the key problem is to construct a probability space rich enough to carry a process that is the limit of finite dimensional approximations.

Loeb measures also allow us to make rigorous the idea of representing arbitrary measures on $\mathbf{H}$ by a measure with a density on $\mathbf{H}_{N}$, and by this means equations for evolving measures can be recast as equations for their evolving nonstandard densities. This theme is developed in Chapters 5 and 7.

As already indicated, in order to make this book self-contained, we have provided a detailed introduction to nonstandard analysis with an emphasis on those parts of this methodology that are needed in this study. This includes a full exposition of Loeb measure theory. 


\section{Results}

We begin in Chapter 4 with an exposition of our nonstandard technique for solving the classical deterministic Navier-Stokes equations, and a discussion of the uniqueness problem using infinitesimals to point up possible sources of non-uniqueness. Starting with the deterministic equations gives a simple illustration of the key ideas of our methods and also provides almost immediately a construction of statistical solutions based on the non-rigorous heuristic originally proposed by Foias (Chapter 5). Our construction utilises a well defined single valued mapping from nonstandard initial conditions to standard solutions - which is lacking in the standard treatment because of possible non-uniqueness (in dimension 3 ). We also present here a new nonstandard treatment of the Hopf formulation of statistical solution, using the idea that in $\mathbf{H}_{N}$ the Hopf equation becomes a linear PDE that can easily be solved.

Loeb space techniques provided the means to solve the general existence problem for stochastic Navier-Stokes equations, which had been open since 1973. This is the main topic of Chapter 6. We give some extensions of our earlier results concerning existence, regularity and uniqueness of solutions. Also new here is a similar treatment of some general equations of thermohydraulics.

We should point out here the differences between our work and earlier pioneering work of Keisler and others in the use of nonstandard techniques to solve stochastic equations. First, Keisler dealt with finite dimensional equations SDE's - whereas here we are infinite dimensional, so we have SPDE's. The other novelty here is that our nonstandard equations are themselves nonstandard SDE's, and to solve them we need to transfer the fully developed theory of finite dimensional SDE's. Keisler's approach was to reduce the standard SDE's to nonstandard stochastic difference equations, which are obviously solvable.

A number of different nonstandard approaches to statistical solutions (in both the Hopf and Foias theories) for stochastic Navier-Stokes equations are possible and are expounded in Chapter 7. These include the approach using nonstandard densities, which allows the construction of statistical solutions (for the stochastic equations) without using any stochastic calculus.

The final chapter deals with the Euler equation, which is significantly more diffcult than the Navier-Stokes equations, but which can be treated by broadly similar nonstandard techniques. 
This page is intentionally left blank 


\section{Contents}

Preface vii

1 Standard Preliminaries $\quad 1$

1.1 Functional Analysis . . . $\quad \ldots \quad \ldots \ldots . \ldots 1$

1.2 Stochastic Analysis . . . . . . . . . . . . . . 5

2 Nonstandard Preliminaries $\quad 11$

2.1 Construction of * $\mathbb{R} \ldots \ldots$

2.2 The Transfer Principle for ${ }^{*} \mathbb{R} \quad \ldots \ldots \ldots$

2.3 Internal Sets and Functions . . . . . . . . . . . . . . 21

2.4 The Hilbert Space $\mathbf{H}$. $\quad \ldots . \quad \ldots \quad \ldots \quad \ldots \quad \ldots 30$

2.5 Countable Comprehension and $\aleph_{1}$-Saturation . . . . . . . . . 31

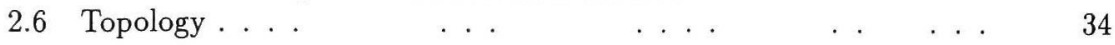

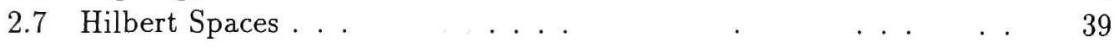

3 Loeb Measure and Integration Theory $\quad 47$

3.1 The Loeb Construction _. . . . . . . . . . . . . . . . . . 47

3.2 Representations of Standard Measures . . . . . . . . . 50

3.3 Loeb Measurable Functions and Integration _. . . . . . . . 54

3.4 Nonstandard Densities of Measures on Hilbert Spaces . . . . . 60

3.5 Bochner Integration . . . . . 64

3.6 Stochastic Integration in Hilbert Spaces . . . . . . . 68

4 Weak Solutions of Navier-Stokes Equations $\quad \mathbf{7 7}$

4.1 Definition of Solutions and Galerkin Approximations . . . . . 78

4.2 Existence of Solutions $\quad \ldots . \quad \ldots \quad$. 79

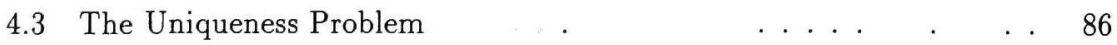

4.4 Notes. $\quad$. . $\quad 92$

5 Statistical Solutions of Navier-Stokes Equations 93

5.1 Space-time Solutions . . . . . . . . . . 95

5.2 Spatial Solutions . . . . . . . . . . . . . . . . 100

5.3 Statistical Solutions Using Nonstandard Densities . . . . . . . 103 
5.4 The Hopf Equation . . . . . . . . . . . . . . . . . . . . . . 111

5.5 Uniqueness of Statistical Solutions . . . . . . . . . . . . . . . 116

5.6 Intrinsic Turbulence. . . . . . . . . . . . . . . . . . 122

5.7 Notes . . . . . . . . . . . . . . . . 129

6 Stochastic Navier-Stokes Equations $\quad 131$

6.1 Definition of Solution . . . . . . . 132

6.2 Solution of a Particular Case . . . . . . . . . . . . 133

6.3 A Special Case of Multiplicative Noise . . . . . . . . . . . . 138

6.4 The General Case: Existence . . . . . . . . . . . . 145

6.5 Regularity of Solutions . . . . . . . . . . . . . . 152

6.6 Uniqueness Theorems . . . . . . . . . . . . . . . . . . 159

6.7 Thermohydraulics . . . . . . . . . . . . . . . 164

6.8 Notes. . . . . . . . . . . . . . . . . . . . 167

7 Statistical Solutions of Stochastic Navier-Stokes Equations $\quad 169$

7.1 The Foias Equation . . . . . . . . . . . 169

7.2 The Hopf Equation . . . . . . . . $\quad$. . . . . . . 173

7.3 Statistical Solutions by Nonstandard Densities . . . . . . . . . 175

7.4 Notes . . . . . . . . . . . . . . . . . . . . . 183

8 The Euler Equation $\quad \mathbf{1 8 5}$

8.1 The General Case . . . . . . . . . . . . . . . . 185

8.2 Two-dimensional Flow on the Torus . . . . . . . . . . 187

8.3 Construction of the Flow and Invariant Measures _ . . . . . . . 191

8.4 Invariant Densities . . . . . . . . . . . . . . . 200

8.5 Statistical Solutions . . . . . . . . . . . . . . . . . . 203

8.6 Notes. . . . . . . . . . . . . . . . . . . 205

$\begin{array}{ll}\text { Appendix } & 207\end{array}$

$\begin{array}{ll}\text { Bibliography } & \mathbf{2 1 7}\end{array}$

$\begin{array}{ll}\text { Notation } & 221\end{array}$

$\begin{array}{lr}\text { Index } & \mathbf{2 2 5}\end{array}$ 